\title{
Gratidão no Contexto Brasileiro: Mensuração e Relações com Personalidade e Bem-Estar
}

\author{
Jean C. Natividade ${ }^{1}$, Nathalia M. Carvalho, Amanda Londero-Santos \\ Thainá F. Carvalho, Leonardo S. Santos, Luiza S. Fagundes \\ Pontifícia Universidade Católica do Rio de Janeiro, Rio de Janeiro-RJ, Brasil
}

\section{RESUMO}

Pesquisas sobre gratidão têm se mostrado cada vez mais frequentes, sobretudo, devido às suas relações com o bem-estar. O objetivo desta pesquisa foi adaptar e buscar evidências de validade para o Brasil de uma medida de gratidão, o Gratitude Questionnaire. Foram realizados procedimentos controlados de tradução e aplicou-se o instrumento em 916 adultos, média de idade de 37 anos, predominantemente com ensino superior completo. Os resultados revelaram adequação dos dados à estrutura de um fator do instrumento, com adequada consistência interna e itens altamente discriminativos. As correlações entre gratidão e demais variáveis mostraram-se de acordo com o esperado teoricamente: positivas com autoestima, otimismo, satisfação de vida e afeto positivo. A gratidão ainda explicou o bem-estar subjetivo para além dos fatores de personalidade. O instrumento mostrou satisfatórias evidências de validade no contexto brasileiro, contudo, observou-se que ele carece de itens que cubram o espectro mais alto do traço latente.

Palavras-chave: gratidão; validade do teste; bem-estar subjetivo; traços de personalidade.

ABSTRACT - Gratitude in the Brazilian Context: Measurement and Relationship with Personality and Well-Being Studies on gratitude are becoming increasingly frequent, mainly because of its relationship with well-being. The aim of this study was to adapt an instrument of gratitude, the Gratitude Questionnaire, and to seek evidence of its validity for Brazil. Controlled translation procedures were performed, and the instrument was applied to 916 adults, mean age 37 years, predominantly with higher education level. The results revealed adequacy of the data for a single factor structure of the instrument, with satisfactory reliability, and highly discriminative items. The correlations between gratitude and other variables were as theoretically expected: Positive with self-esteem, optimism, life satisfaction, and positive affect. Gratitude also explained subjective well-being beyond personality factors. The instrument showed satisfactory evidence of validity in the Brazilian context; however, it was observed that it lacks items that cover the higher spectrum of the latent trait.

Keywords: gratitude; test validity; subjective well-being; personality traits.

\section{RESUMEN - Gratitud en el Contexto Brasileño: Medición y Relación con la Personalidad y el Bienestar}

Las investigaciones sobre la gratitud han sido cada vez más comunes, especialmente debido a sus relaciones con el bienestar. El objetivo de esta investigación fue adaptar y buscar evidencias de validez de un instrumento de gratitud, el Gratitude Questionnaire, para ser utilizado en Brasil. Se realizaron procedimientos de traducción controlados y se aplicó el instrumento a 916 adultos, con una edad media de 37 años, predominantemente con un nivel de educación superior. Los resultados revelaron la adecuación de los datos a la estructura de un factor del instrumento, con confiabilidad satisfactoria e ítems altamente discriminantes. Las correlaciones entre la gratitud y las demás variables fueron las esperadas: positivas con autoestima, optimismo, satisfacción con la vida, y afecto positivo. La gratitud también explica el bienestar subjetivo más allá de los factores de personalidad. El instrumento presentó satisfactoria evidencia de validez en el contexto brasileño; sin embargo, se observó que carece de elementos que cubran el espectro más alto del rasgo latente.

Palabras clave: gratitud; validez de test; bienestar subjetivo; rasgos de personalidad.

As pesquisas sobre gratidão na psicologia podem ser consideradas recentes, ainda que esse tema já venha sendo alvo de estudos de disciplinas como a filosofia e a teologia há longo tempo (Emmons \& Shelton, 2002). No início dos anos 2000, McCullough, Emmons e Tsang (2002) publicaram uma das pesquisas pioneiras sobre gratidão. Nesse estudo, os autores apresentaram uma medida de gratidão, o Gratitude Questionnaire (GQ-6). Desde então, a gratidão se estabeleceu como uma variável importante no campo da Psicologia Positiva. Estudos nessa área têm mostrado que pessoas gratas tendem a serem mais felizes e mais saudáveis (Wood, Froh, \& Geraghty, 2010). Apesar disso, poucos estudos propuseram-se a medir a gratidão no Brasil. Em vista disso, delineou-se esta pesquisa com os objetivos de adaptar e buscar evidências de validade do GQ-6 para o contexto brasileiro, bem como de testar 
relações entre gratidão, autoestima, otimismo, bem-estar subjetivo e os cinco grandes fatores de personalidade.

\section{O Gratitude Questionnaire (GQ-6)}

A gratidão já foi entendida de diversas formas, por exemplo, como um hábito, uma virtude e uma característica individual (Emmons \& Shelton, 2002). Na Psicologia, a compreensão da gratidão como um traço afetivo tem sido bem aceita (e.g., Emmons \& Shelton, 2002; McCullough et al., 2002). Os traços afetivos indicam diferentes padrões na forma como as pessoas tendem a reagir emocionalmente aos eventos de vida. Esses padrões variam pouco em função do tempo e do ambiente e orientam uma ampla gama de comportamentos (McCullough et al., 2002). No caso da gratidão, uma pessoa que demonstra uma predisposição para se sentir grata, provavelmente, sentir-se-á grata ao longo de mais dias e diante de mais estímulos eliciadores (McCullough et al., 2002).

McCullough et al. (2002) partiram da perspectiva da gratidão como um traço afetivo para construir o GQ-6. Segundo esses autores, diferenças individuais nos níveis de gratidão podem ser sistematizadas em um construto unidimensional chamado disposição frente à gratidão, que indica uma tendência generalizada a sentir-se grato diante de acontecimentos positivos. Em indivíduos que possuem essa predisposição, a gratidão surgiria como resposta emocional ao reconhecimento de que outras pessoas teriam contribuído para resultados positivos obtidos. Indivíduos mais gratos tendem a experimentar essa emoção mais intensamente e com maior frequência, além de reconhecerem mais motivos pelos quais agradecer e mais pessoas às quais atribuir algum nível de responsabilidade por um resultado positivo. Essas características são chamadas de intensidade, frequência, alcance, densidade, e constituem a gratidão (McCullough et al., 2002).

No GQ-6, a disposição para se sentir grato é medida por meio de seis itens de autorrelato. Os participantes são solicitados a responder o quanto concordam com as afirmativas em uma escala de sete pontos. Quanto maior o escore obtido na escala, maior o nível de gratidão experimentado, tanto em termos de intensidade e frequência, quanto de alcance e densidade. No estudo de construção do instrumento, o GQ-6 apresentou adequadas evidências de validade, incluindo estrutura unifatorial, consonante com a definição do construto, e alta consistência interna. Além disso, a gratidão se correlacionou positivamente com vitalidade, esperança, emoções positivas e satisfação de vida, e negativamente com ansiedade e depressão, conforme esperado (McCullough et al., 2002). Esse instrumento foi elaborado para adultos no contexto norte-americano e adaptado para outras culturas, como Chile (Langer, Ulloa, Aguilar-Parra, Araya-Véliz, \& Brito, 2016), China (Kong, You, \& Zhao, 2017), Itália (Caputo, 2016), Taiwan (Chen, Chen, Kee, \& Tsai, 2009).
No contexto brasileiro, a primeira versão adaptada do GQ-6 apresentou uma estrutura de dois fatores, diferentemente da escala original (ver Paludo, 2008). Esse resultado pode ter sido provocado, sobretudo, pelas características da população estudada: crianças e adolescentes de baixa renda da região sul do país. Quando uma versão brasileira do GQ-6 foi aplicada em uma amostra de adultos, Natividade (2018) encontrou uma estrutura unifatorial para a escala, consonante com o estudo original, e relações esperadas entre gratidão e outros construtos positivos, como autoestima e otimismo. Em outra adaptação do GQ-6 para o contexto brasileiro, Gouveia et al. (2019) testaram a estrutura do instrumento em duas amostras de jovens universitários da região Nordeste. Entre as evidências de validade encontradas, destacam-se a estrutura de um único fator e a correlação positiva de gratidão com sentido de vida.

Apesar dos resultados satisfatórios no que diz respeito à utilização do GQ-6 no Brasil, novas evidências de validade são desejáveis, visto que a validação de instrumentos psicológicos é considerada um processo contínuo e cumulativo (American Educational Research Association [AERA], American Psychological Association [APA], \& National Council on Measurement in Education [NCME], 2014). Neste estudo, buscou-se ampliar o conjunto de evidências de validade existentes até o momento no Brasil, por exemplo, utilizando-se participantes de todas as regiões do país, investigando-se os parâmetros de discriminação (slope) e dificuldade (threshold) dos itens e testando-se relações entre gratidão, bem-estar subjetivo e os cinco grandes fatores de personalidade.

\section{Gratidão, Bem-estar e Personalidade}

O interesse mais recente pelo estudo da gratidão decorre, principalmente, das evidências que apontam para uma forte relação entre gratidão e bem-estar (Wood et al., 2010). Diener, Oishi e Tay (2018) definem bem-estar subjetivo como uma avaliação subjetiva das pessoas sobre a própria vida, incluindo julgamentos cognitivos (satisfação de vida) e experiências emocionais positivas ou negativas (afetos positivo e negativo). Altos níveis de gratidão indicam uma tendência a apresentar níveis mais elevados de satisfação de vida e afeto positivo, e níveis mais baixos de afeto negativo (e.g., Emmons \& McCullough, 2003; McCullough, Tsang, \& Emmons, 2004; Park, Peterson, \& Seligman, 2004; Wood et al., 2010). Além disso, agradecer diariamente por eventos de vida positivos, por exemplo, mostra-se um exercício eficaz para melhorar diversos indicadores de saúde, como a satisfação de vida, as emoções positivas e o hábito de praticar exercícios físicos regularmente (Emmons \& McCullough, 2003).

No que diz respeito à personalidade, a gratidão tem mostrado o mesmo padrão de associações que o bem-estar. Os estudos sobre gratidão e personalidade partem do modelo dos cinco grandes fatores de personalidade, ou Big5. O Big5 tem se mostrado replicável em diversas 
culturas e abarca os principais aspectos da personalidade humana (Natividade \& Hutz, 2015). Estudos que se basearam nesse modelo mostram correlações positivas entre a gratidão e os seguintes fatores: extroversão, uma tendência a buscar estimulação social; socialização, uma tendência a demonstrar empatia, comportamento pró-social; realização, uma tendência a ser disciplinado para cumprir metas; e abertura, uma tendência a buscar novas experiências. Correlação negativa foi encontrada entre gratidão e neuroticismo, o que é esperado, pois esse fator indica uma tendência a vivenciar emoções negativas e instabilidade emocional (McCullough et al., 2004; Wood, Joseph, \& Malbty, 2008; Wood, Maltby et al., 2008; Wood, Maltby, Stewart, \& Joseph, 2008).

A gratidão e o bem-estar, portanto, correlacionam-se com os cinco grandes fatores nas mesmas direções: positivamente com os traços associados a um funcionamento saudável e negativamente com neuroticismo (McCullough et al., 2004; Woyciekoski, Natividade, \& Hutz, 2014). Por esse motivo, segundo alguns autores, não bastaria mostrar que a gratidão e o bem-estar subjetivo tendem a variar juntos, pois essa relação poderia existir apenas em função de uma terceira variável; seria preciso avaliar em que medida a gratidão teria impactos significativos nos níveis de bem-estar, para além dos cinco grandes fatores (McCullough et al., 2002; Wood, Joseph, \& Maltby, 2009). Se o poder preditivo da gratidão sobre outras variáveis puder ser inteiramente explicado em termos da sua variância compartilhada com fatores mais amplos de personalidade (e.g., neuroticismo, socialização, extroversão), então esta deveria ser estudada como uma manifestação de traços mais fundamentais, não como um construto diferenciado (McCullough et al., 2002; Wood et al., 2009).

Evidências empíricas a esse respeito apontam para uma relação significativa entre gratidão e bem-estar, independentemente da personalidade. Em modelos preditivos, a gratidão explicou a variação da satisfação de vida para além dos cinco grandes fatores (Wood et al., 2008), e isso foi encontrado também em relação ao bem-estar psicológico (Wood et al., 2009). Nessa mesma direção, outros estudos mostraram que as relações entre a gratidão e os três componentes do bem-estar subjetivo continuam a existir mesmo quando os efeitos da personalidade são controlados (McCullough et al., 2002; Wood, Maltby, Gillett, Linley, \& Joseph, 2008). Além disso, os cinco grandes fatores explicam em torno de $21 \%$ da variância da gratidão (McCullough et al., 2002) e as correlações encontradas entre a gratidão e outros traços, como o otimismo, são apenas moderadas (McCullough et al., 2002).

Todos juntos, esses resultados sugerem que a gratidão pode ser considerada um construto psicológico independente, com poder explicativo único sobre o bem-estar (McCullough et al., 2002; Wood et al., 2009). Embora a gratidão compartilhe variância com os cinco grandes fatores de personalidade, o bem-estar subjetivo, o otimismo, a autoestima e outras variáveis positivas, como a esperança e a prossociabilidade, há evidências de que esses construtos seriam apenas correlacionados, não equivalentes (McCullough et al., 2002). Essa compreensão possibilitou avanços em psicologia positiva, como a elaboração de técnicas aplicáveis ao contexto clínico e a busca por um melhor entendimento sobre as variáveis que atuariam como preditoras de níveis disposicionais de gratidão (e.g., Emmons \& McCullough, 2003).

\section{Gratidão e Variáveis Sociodemográficas}

O interesse em estimular a gratidão para promover o bem-estar levou alguns pesquisadores a se questionarem sobre o perfil sociodemográfico de indivíduos mais predispostos a se sentirem gratos, assim como daqueles que raramente experimentam essa emoção (e.g., Allemand \& Hill, 2016). Uma primeira questão de interesse consistiu em identificar possíveis diferenças sexuais nos níveis de gratidão, considerando que homens e mulheres experimentam emoções de maneira distinta (Naito, Wangwan, \& Tani, 2005; Simon \& Nath, 2004). Nesse sentido, alguns estudos mostram que as mulheres sentem e expressam mais gratidão, além de atribuírem um maior número de benefícios a essa emoção (Kashdan, Mishra, Been, \& Froh, 2009). Por outro lado, os homens mostram-se com uma postura mais crítica e contida em relação à gratidão, possivelmente porque senti-la e demonstrá-la provoque desconforto (Kashdan et al., 2009). Enquanto as mulheres se sentiriam entusiasmadas com o vínculo emocional provocado pela gratidão, homens se sentiriam vulneráveis, acreditando que possuem uma dívida a ser paga com o benfeitor (Kashdan et al., 2009).

Outra variável que se mostra associada à gratidão de maneira significativa é a religiosidade - uma relação que remete aos primórdios dos estudos sobre gratidão, quando ainda não havia métodos para acessá-la empiricamente. Já nessa época, grupos religiosos como o cristianismo, o judaísmo e o islã incentivavam os fiéis a darem graças pelas bênçãos recebidas e enfatizavam a gratidão como uma qualidade de pessoas virtuosas, ou um hábito que conduziria a uma vida plena (Emmons, 2005). Uma relação entre gratidão e religiosidade é esperada por alguns motivos. Primeiro, indivíduos que participam ativamente de rituais religiosos estariam mais expostos a discursos que encorajariam a gratidão. Além disso, pode ser que indivíduos mais gratos sejam mais inclinados à espiritualidade (McCullough et al., 2002). Quando essas pessoas se veem diante de resultados positivos que não estão associados a uma ação humana, por exemplo, um belo dia de sol, tenderiam a se sentir gratas a um agente sobrenatural, como um deus ou uma força superior (McCullough et al., 2002).

Evidências empíricas têm confirmado relações entre diversas dimensões de religiosidade e gratidão (e.g., Lambert, Fincham, Braithwaite, Graham, \& Beach, 
2009; McCullough et al. 2004). Pessoas que rezam mais, frequentam a igreja mais vezes e possuem mais amigos religiosos tendem a demonstrar níveis mais elevados de gratidão (Adler \& Fagley, 2005; Lambert et al., 2009; Kraus, Desmond, \& Palmer, 2015). Em comparação a um grupo controle, um grupo de pessoas que foi instruído a rezar diariamente apresentou níveis maiores de gratidão após um período de seis semanas (Lambert et al., 2009). Além disso, pessoas que percebem que suas preces são atendidas, por exemplo, ao experienciarem respostas divinas ou milagres, tendem a ser mais gratas (Kraus et al., 2015). Mesmo depois de controlar os efeitos de outras variáveis, as relações entre gratidão e religiosidade se mantêm, indicando que pessoas religiosas tendem a ser mais gratas independentemente do sexo, da idade e da escolaridade (Krause, 2009).

Em relação a possíveis variações nos níveis de gratidão ao longo da vida adulta, os estudos têm mostrado que pessoas mais velhas teriam uma tendência maior a sentir gratidão e isso se daria devido ao processo de amadurecimento (Chopik, Newton, Ryan, Kashdan, \& Jarden, 2019; Kashdan et al., 2009). No entanto, outros autores defendem o uso de indicadores mais subjetivos para mensurar a passagem do tempo, além da idade cronológica (Allemand \& Hill, 2016). Quando isso foi feito, a gratidão se mostrou maior em indivíduos que percebem seu tempo futuro como em aberto, repleto de oportunidades e possibilidades, em comparação a indivíduos que acreditam possuir um tempo de vida limitado (Allemand \& Hill, 2016).

\section{Presente Estudo}

Considerando-se a necessidade de constante busca por evidências de validade para os instrumentos psicológicos, delineou-se este estudo com os objetivos de adaptar o GQ-6 para o contexto brasileiro e buscar evidências de validade e precisão dessa versão adaptada. Mais especificamente, buscaram-se evidências de validade baseadas no conteúdo, na estrutura interna e nas relações com outras variáveis. Foram testadas relações entre gratidão, personalidade, bem-estar subjetivo, otimismo, autoestima e idade, diferenças nos níveis de gratidão entre homens e mulheres e, ainda, diferenças nos níveis de gratidão entre pessoas que possuem e pessoas que não possuem uma crença religiosa. Além disso, testou-se o poder preditivo da gratidão sobre o bem-estar subjetivo para além dos cinco grandes fatores de personalidade.

\section{Método}

\section{Participantes}

Participaram 916 adultos brasileiros, média de idade de 37,0 anos $(D P=14,3)$, sendo 69,9\% mulheres. Houve participantes das cinco regiões do Brasil, tal que 72,4\% eram do Sudeste; $13,4 \%$, do Sul; 7,64\%, do Centro-oeste; $3,17 \%$, do Nordeste; $1,75 \%$, do Norte; e os demais 1,64\% não estavam no Brasil no momento da coleta de dados. Quanto à escolaridade, 68,6\% dos participantes tinham ensino superior completo (sendo que $36,7 \%$ do total de participantes também tinham pós-graduação completa), $26,6 \%$ tinham ensino superior incompleto, e os demais $4,8 \%$ tinham até ensino médio completo. No que diz respeito à raça/cor, 75,8\% dos participantes declararam-se brancos; $16,9 \%$, pardos; $3,5 \%$, pretos; $1,9 \%$, amarelos; $0,3 \%$, indígenas; e $1,6 \%$ não sabia ou não queria informar. Em relação a possuir uma crença religiosa, 75,5\% dos participantes afirmaram acreditar em algum deus ou força espiritual.

\section{Instrumentos}

Utilizou-se um questionário on-line, disponibilizado em um endereço na internet, contendo perguntas sociodemográficas, tais como: sexo, idade, escolaridade, raça/cor, cidade em que residia, se tinha alguma crença religiosa/religião. Além dessas perguntas, o questionário continha instrumentos para aferir a gratidão, autoestima, otimismo, bem-estar subjetivo (satisfação de vida e afetos positivo e negativo) e os cinco grandes fatores de personalidade. O questionário foi configurado para não permitir respostas omissas aos itens da escala de gratidão.

Gratitude Questionnaire - GQ-6 (McCullough et al., 2002; versão adaptada para o contexto brasileiro neste estudo). Esse instrumento, unifatorial, mede a disposição frente a sentir-se grato diante de situações e pessoas, uma tendência generalizada a sentir gratidão. Trata-se de uma escala composta por seis itens em formato de afirmativas em que as pessoas devem indicar o quanto concordam com as afirmativas em uma escala de sete pontos. Quanto maiores os escores obtidos na escala, maiores os níveis de gratidão. $\mathrm{O}$ instrumento apresentou coeficiente alfa de 0,82 na versão original de McCulloug et al. Nessa escala, incluiu-se um item controle em que se solicitava aos participantes que respondessem de determinada maneira (e.g., marcando o número cinco como resposta).

Escala de Autoestima de Rosenberg (Hutz \& Zanon, 2011; versão brasileira do original de Rosenberg, 1965). Trata-se de um instrumento unifatorial constituído por 10 afirmações para aferir a autoestima global, uma atitude sobre o autoconceito. Os itens são respondidos em uma escala de quatro pontos. Quanto maiores os escores obtidos na escala, maiores os níveis de autoestima. No estudo de Hutz e Zanon, a escala apresentou coeficiente alfa de 0,90 .

Teste de Orientação da Vida Revisado - LOT-R (Bastianello \& Pacico, 2014; versão brasileira do original de Scheier, Carver, \& Bridges, 1994). Esse instrumento, unifatorial, avalia o otimismo disposicional, expectativas positivas generalizadas em relação a eventos futuros. O instrumento é composto por dez itens, sendo quatro distratores, em formato de afirmativas a serem respondidas em uma escala de concordância de cinco pontos. Quanto maiores os escores na escala, maiores os níveis 
de otimismo. No estudo de Bastianello e Pacico a escala apresentou coeficiente alfa de 0,80 .

Escala de Satisfação de Vida - ESV (Hutz, Zanon, \& Bardagi, 2014; versão brasileira do original de Diener, Emmons, Larsen, \& Griffin, 1985). Essa escala, unifatorial, afere o aspecto cognitivo do bem-estar subjetivo, um julgamento sobre o quanto a vida está próxima do ideal. É uma escala composta por cinco itens em formato de afirmativas, na qual os participantes devem responder o quanto concordam com cada uma delas, em uma escala de cinco pontos. Quanto maiores os valores obtidos na escala, maior a satisfação de vida do participante. Não é apresentado o valor de coeficiente alfa, no estudo de Hutz et al. (2014).

Escala de Afetos Positivo e Negativo - PANAS (Zanon \& Hutz, 2014; versão brasileira da escala de Watson, Clark, \& Tellegen, 1988). Essa escala acessa os dois fatores afetivos do bem-estar subjetivo: afeto positivo e afeto negativo. Cada fator é composto por 10 adjetivos que representam humores e emoções dos participantes. Os participantes devem responder, em uma escala de cinco pontos, o quanto os adjetivos representam como eles têm se sentindo ultimamente. Quanto maiores os escores em cada fator, maiores os níveis dos afetos. Não são apresentados os valores de coeficientes alfa, no estudo de Zanon e Hutz.

\section{Escala Reduzida de Descritores de} Personalidade - RED5 (Natividade \& Hutz, 2015). Essa escala avalia os cinco grandes fatores de personalidade: extroversão, socialização, realização, neuroticismo, abertura a experiências. A escala é composta de 20 adjetivos ou pequenas expressões, quatro para cada fator, para que os participantes respondam o quanto concordam que cada um deles os descreve adequadamente, em uma escala de sete pontos. Quanto maiores os escores dos participantes em cada fator, maior a intensidade dos fatores. No estudo de Natividade e Hutz, os coeficientes alfa dos fatores variaram de 0,59 a 0,84 e as correlações teste-reteste variaram de 0,69 a 0,81 .

\section{Procedimentos}

De tradução. Inicialmente, o instrumento foi traduzido do inglês para o português, independentemente, por dois pesquisadores bilíngues português-inglês. Em seguida, um terceiro pesquisador bilíngue português-inglês, e com experiência em adaptação de instrumentos psicológicos, comparou as duas versões com o instrumento original em inglês e chegou a uma versão beta. Essa versão beta foi apresentada a três pessoas bilíngues português-inglês alheias à pesquisa, juntamente com a versão original do instrumento em inglês, para que julgassem a adequação da redação dos itens. Baseando-se nas sugestões dessa última avaliação, foram feitos pequenos ajustes para tornar mais clara a redação em português, chegando-se à versão final traduzida.
De coleta. A coleta de dados foi realizada on-line, via internet. $\mathrm{O}$ recrutamento dos participantes deu-se por meio de convites, contendo o endereço do questionário, disponibilizados em redes sociais e no site do laboratório, bem como enviados por e-mail e aplicativos de mensagens. Também se utilizou um recrutamento presencial para coleta on-line nas dependências da universidade (ver Wachelke, Natividade, Andrade, Wolter, \& Camargo, 2014). Nesse recrutamento, coletavam-se os endereços de e-mail daqueles que aceitavam participar da pesquisa e, posteriormente, enviavam-se os convites com endereço do questionário. Esta pesquisa obteve aprovação do Comitê de Ética em Pesquisa com Seres Humanos do Hospital Federal de Ipanema, sob o número de protocolo 3415129.

De análises. Inicialmente, procedeu-se a limpeza dos dados, eliminando-se as respostas erradas à pergunta controle, a fim de eliminar possíveis respostas falseadas. Em seguida, em busca de evidências de validade para o instrumento, testou-se o ajuste dos dados à estrutura unifatorial. Para tanto, realizou-se uma análise fatorial confirmatória por meio do software R versão 3.5.3 (R Core Team, 2019) e do pacote Lavaan versão 0.6.3 (Rosseel, 2012). Nessa análise partiu-se da matriz de covariância e utilizou-se o estimador Maximum Likelyhood Robusto. Também se calcularam os parâmetros de dificuldade e discriminação dos itens por meio do Modelo de Resposta Gradual da teoria de resposta ao item (GRM; Samejima, 1969), utilizando-se o pacote Mirt versão 1.30, no R (Chalmers, 2012). Em seguida, calcularam-se os coeficientes alfa e ômega, a fim de obter indicadores de fidedignidade, utilizando-se o pacote MBESS versão 4.6, no R (Kelley, 2019). Ainda em busca de evidências de validade para a escala, testaram-se correlações de Pearson entre gratidão, autoestima, otimismo, satisfação de vida, afetos positivo e negativo e os cinco grandes fatores de personalidade. Depois, utilizou-se o teste qui-quadrado para testar associação entre sexo e crença religiosa. No que diz respeito às diferenças entre grupos, testaram-se diferenças de médias em gratidão entre quem tinha e quem não tinha uma crença religiosa e entre homens e mulheres, por meio de ANOVAs two-way, a fim de identificar uma possível interação entre essas variáveis. Também se testaram essas mesmas diferenças controlando-se os efeitos dos cinco grandes fatores de personalidade, por meio de uma ANCOVA. Por fim, testou-se o poder preditivo da gratidão sobre o bem-estar subjetivo, controlando-se os efeitos dos cinco grandes fatores, por meio de regressões múltiplas hierárquicas, método Enter.

\section{Resultados}

O modelo especificado com um fator explicando todos os seis itens da gratidão obteve os seguintes índices de ajuste: $\chi^{2}(9, N=916)=59,9 ; p<0,001 ; \chi^{2} /$ $g l=6,66$; Tucker-Lewis Index (TLI)=0,93; Comparative Fit 
Index $(\mathrm{CFI})=0,96 ;$ Goodness-of-Fit Index $(\mathrm{GFI})=0,97$; Root Mean Square Error of Aproximation (RMSEA) $=0,094$ $(\mathrm{CI} 90 \%=0,072-0,12)$. Observando-se os índices de modificação, configurou-se o modelo de um fator correlacionando-se os resíduos de dois itens da escala: itens 3 e 6. Esse modelo com os resíduos correlacionados obteve os seguintes índices de ajuste: $\chi^{2}(8, N=916)=31,6$; $p<0,001 ; \chi^{2} / g l=3,95 ; \mathrm{TLI}=0,96 ; \mathrm{CFI}=0,98 ; \mathrm{GFI}=0,98$; RMSEA $=0,067 \quad(\mathrm{CI} 90 \%=0,044-0,093)$. Os resultados sugerem adequação da estrutura unifatorial da escala. As cargas fatoriais e os parâmetros dos itens podem ser vistos na Tabela 1.

Tabela 1

Cargas Fatoriais e Parâmetros de Discriminação e Dificuldade dos Itens

\begin{tabular}{lccccccccc}
\hline & $\lambda$ & $a$ & $b 1$ & $b 2$ & $b 3$ & $b 4$ & $b 5$ & $b 6$ \\
\hline Item 1 & 0,75 & 3,45 & $-3,03$ & $-2,64$ & $-2,25$ & $-1,88$ & $-1,34$ & $-0,46$ \\
Item 2 & 0,84 & 3,31 & $-2,82$ & $-2,17$ & $-1,74$ & $-1,30$ & $-0,89$ & 0,05 \\
Item 3 & $-0,56$ & 1,76 & $-3,02$ & $-2,22$ & $-1,76$ & $-1,51$ & $-1,11$ & $-0,17$ \\
Item 4 & 0,66 & 1,87 & $-3,31$ & $-2,23$ & $-1,80$ & $-1,41$ & $-0,77$ & 0,35 \\
Item 5 & 0,54 & 1,32 & $-4,45$ & $-3,37$ & $-2,91$ & $-2,28$ & $-1,53$ & $-0,07$ \\
Item 6 & $-0,45$ & 1,13 & $-3,89$ & $-2,68$ & $-1,84$ & $-1,20$ & $-0,70$ & 0,69 \\
\hline
\end{tabular}

Nota. $\lambda=$ carga fatorial. a=parâmetro de discriminação/slope. $b=$ parâmetros de dificuldade/threshold. Cargas fatoriais obtidas por meio de análise fatorial confirmatória, método Maximum Likelyhood Robusto. Parâmetros de discriminação e dificuldade estimados pelo Graded Response Model (Samejima, 1969). Os itens 3 e 6 foram invertidos para o cálculo dos parâmetros

O teste apresentou coeficiente alfa e ômega de 0,79 , com valores entre 0,76 a 0,81 para um intervalo de confiança de 95\%, 500 amostras bootstrap. Adicionalmente, elaborou-se a curva de informação do teste, ver Figura 1.

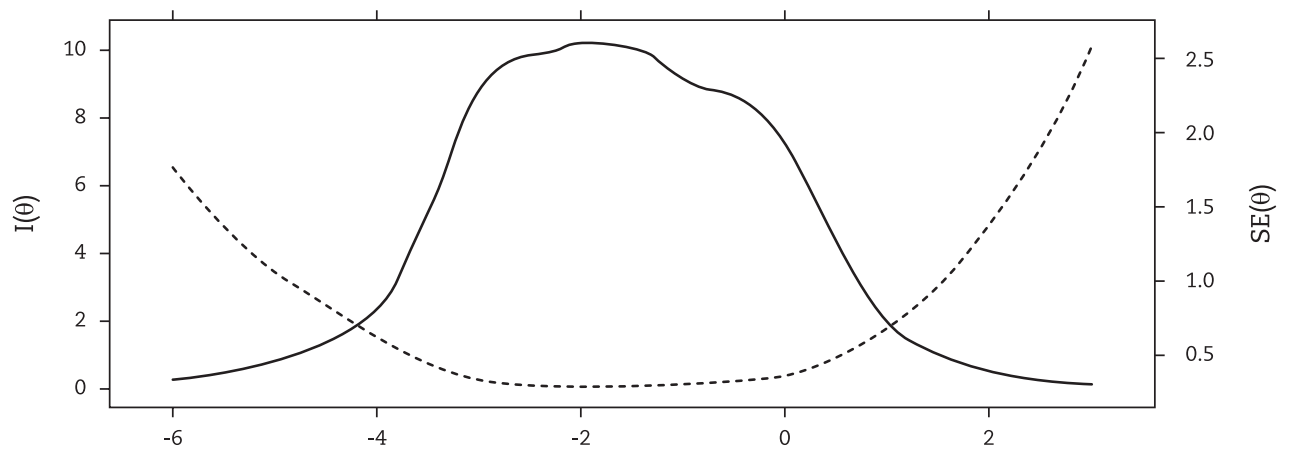

$(\theta)$

Figura 1. Curva de informação do teste. A linha contínua representa a curva de informação do teste. A linha pontilhada representa o erro padrão da medida

Em busca de evidências de validade baseadas nas relações com outras variáveis, testaram-se correlações entre gratidão, autoestima, otimismo, bem-estar subjetivo e personalidade. Os coeficientes de correlação de Pearson podem ser vistos na Tabela 2. Também se verificou que o coeficiente de correlação múltipla entre gratidão e os cinco grandes fatores de personalidade foi de $R=0,54$.

O teste qui-quadrado revelou uma associação entre sexo e crença religiosa, sendo que $80,6 \%$ das mulheres afirmaram ter uma religião, enquanto $63,8 \%$ dos homens afirmaram isso, $\chi^{2}(1, N=916)=29,7 ; p<0,001$. Por esse motivo, realizou-se uma ANOVA two-way para testar o efeito do sexo e de ter uma crença religiosa sobre os níveis de gratidão. Não foi verificada interação entre os efeitos dessas duas variáveis sobre a gratidão, $F(1,912)=0,191$; $p=0,66$. No entanto, tanto sexo, quanto crença religiosa apresentaram efeitos isolados sobre a gratidão. Verificouse que as mulheres $(n=640 ; M=6,12 ; D P=0,88)$ mostraram maiores níveis de gratidão do que os homens $(n=276$; $M=5,64 ; D P=1,03), F(1,912)=25,9 ; p<0,001 ; d=0,39$. $\mathrm{E}$ também que as pessoas que afirmaram ter uma crença religiosa $(n=692 ; \mathrm{M}=6,15 ; \mathrm{DP}=0,81)$ mostraram maiores níveis de gratidão do que quem não tinha crença 
religiosa $(n=224 ; M=5,45 ; \mathrm{DP}=1,15), F(1,912)=75,5$; $p<0,001, d=0,69$.

Adicionalmente, testaram-se essas mesmas diferenças sexuais e de crença religiosa na gratidão controlando-se os efeitos dos cinco grandes fatores de personalidade, por meio de uma ANCOVA. Os resultados foram muito semelhantes aos anteriores. Não foi verificada interação entre sexo e crença religiosa sobre os níveis de gratidão, $F(1,874)=1,24 ; p=0,27$; e foram encontradas diferenças em gratidão entre homens e mulheres, $F(1,874)=19,3$; $p<0,001$, e entre quem tinha uma crença religiosa e quem não tinha, $F(1,874)=43,6 ; p<0,001$, sendo as mulheres e aqueles com crença religiosa os com maiores níveis de gratidão.

Tabela 2

Coeficientes de Correlação de Pearson entre Gratidão, Autoestima, Otimismo, Bem-estar subjetivo, Personalidade e Idade

\begin{tabular}{lccccccccccc}
\hline & 1 & 2 & 3 & 4 & 5 & 6 & 7 & 8 & 9 & 10 & 11 \\
\hline 1. Gratidão & $(0,79)$ & & & & & & & & & & \\
2. Autoestima, $N=893$ & $0,52^{* *}$ & $(0,90)$ & & & & & & & & & \\
3. Otimismo, $N=893$ & $0,54^{* *}$ & $0,72^{* *}$ & $(0,85)$ & & & & & & & & \\
4. Satisfação de vida & $0,55^{* *}$ & $0,63^{* *}$ & $0,54^{* *}$ & $(0,88)$ & & & & & & & \\
5. Afeto Positivo & $0,53^{* *}$ & $0,64^{* *}$ & $0,57^{* *}$ & $0,60^{* *}$ & $(0,88)$ & & & & & & \\
6. Afeto Negativo & $-0,36^{* *}$ & $-0,58^{* *}$ & $-0,51^{* *}$ & $-0,49^{* *}$ & $-0,45^{* *}$ & $(0,88)$ & & & & & \\
7. Extroversão, $N=883$ & $0,39^{* *}$ & $0,38^{* *}$ & $0,37^{* *}$ & $0,25^{* *}$ & $0,39^{* *}$ & $-0,18^{* *}$ & $(0,86)$ & & & & \\
8. Socialização, $N=883$ & $0,42^{* *}$ & $0,37^{* *}$ & $0,39^{* *}$ & $0,27^{* *}$ & $0,37^{* *}$ & $-0,28^{* *}$ & $0,55^{* *}$ & $(0,81)$ & & & \\
9. Realização, $N=883$ & $0,32^{* *}$ & $0,41^{* *}$ & $0,30^{* *}$ & $0,33^{* *}$ & $0,35^{* *}$ & $-0,29^{* *}$ & $0,14^{* *}$ & $0,19^{* *}$ & $(0,71)$ & & \\
10. Neuroticismo, $N=883$ & $-0,29^{* *}$ & $-0,55^{* *}$ & $-0,47^{* *}$ & $-0,37^{* *}$ & $-0,35^{* *}$ & $0,62^{* *}$ & $-0,10^{* *}$ & $-0,27^{* *}$ & $-0,28^{* *}$ & $(0,68)$ & \\
11. Abertura, $N=883$ & $0,19^{* *}$ & $0,24^{* *}$ & $0,25^{* *}$ & $0,14^{* *}$ & $0,33^{* *}$ & $-0,09^{* *}$ & $0,29^{* *}$ & $0,23^{* *}$ & 0,01 & $-0,16^{* *}$ & $(0,59)$ \\
12. Idade & $0,27^{* *}$ & $0,37^{* *}$ & $0,31^{* *}$ & $0,18^{* *}$ & $0,15^{* *}$ & $-0,24^{* *}$ & $0,20^{* *}$ & $0,11^{* *}$ & $0,27^{* *}$ & $-0,26^{* *}$ & 0,05 \\
\hline
\end{tabular}

Nota. Quando não indicado, $N=916$. Coeficientes alfa das escalas na diagonal principal, entre parênteses. ${ }^{* *} p<0,01$

Por fim, testou-se o poder preditivo da gratidão sobre o bem-estar subjetivo, buscando-se identificar se a gratidão explicaria o bem-estar para além da personalidade. Realizaram-se três análises de regressão hierárquica, uma para cada componente do bem-estar subjetivo, em que se incluíram no primeiro bloco os fatores de personalidade e, no segundo, a gratidão. Os resultados podem ser vistos nas Tabelas 3, 4 e 5. A gratidão entrou como variável explicativa significativa em todos os três modelos testados (satisfação de vida, afeto positivo, afeto negativo), destacando-se o incremento de $13 \%$ na explicação da satisfação de vida e $7 \%$ do afeto positivo após a inclusão da gratidão nos modelos.

Tabela 3

Personalidade e Gratidão como Preditoras da Satisfação de Vida

\begin{tabular}{|c|c|c|c|c|c|c|}
\hline & \multicolumn{3}{|c|}{ Bloco 1} & \multicolumn{3}{|c|}{ Bloco 2} \\
\hline & $\beta$ & $t$ & $p$ & $\beta$ & $t$ & $p$ \\
\hline Constante & & 6,39 & $<0,001$ & & 1,45 & 0,15 \\
\hline Extroversão & 0,15 & 4,10 & $<0,001$ & 0,06 & 1,65 & 0,10 \\
\hline Socialização & 0,07 & 1,88 & 0,061 & $-0,02$ & $-0,58$ & 0,56 \\
\hline Realização & 0,22 & 7,18 & $<0,001$ & 0,14 & 4,64 & $<0,001$ \\
\hline Neuroticismo & $-0,27$ & $-8,30$ & $<0,001$ & $-0,20$ & $-6,86$ & $<0,001$ \\
\hline Abertura & 0,03 & 1,08 & 0,28 & 0,01 & 0,47 & 0,64 \\
\hline Gratidão & & & & 0,43 & 13,4 & $<0,001$ \\
\hline$R^{2}$ & & 0,23 & & & 0,36 & \\
\hline$R^{2}$ ajustado & & 0,23 & & & 0,36 & \\
\hline$F$ & & $53,3^{* * *}$ & & & $83,2^{* * *}$ & \\
\hline
\end{tabular}

Nota. $\mathrm{N}=883$. Regressão linear hierárquica, método Enter, fatores de personalidade no primeiro bloco e gratidão incluída no segundo bloco. ${ }^{* * *} \mathrm{p}<0,001$ 
Tabela 4

Personalidade e Gratidão como Preditoras do Afeto Positivo.

\begin{tabular}{|c|c|c|c|c|c|c|}
\hline & \multicolumn{3}{|c|}{ Bloco 1} & \multicolumn{3}{|c|}{ Bloco 2} \\
\hline & $\beta$ & $t$ & $p$ & $\beta$ & $t$ & $p$ \\
\hline Constante & & 4,94 & $<0,001$ & & 0,95 & 0,34 \\
\hline Extroversão & 0,22 & 6,62 & $<0,001$ & 0,15 & 4,72 & $<0,001$ \\
\hline Socialização & 0,10 & 2,96 & 0,003 & 0,03 & 1,05 & 0,29 \\
\hline Realização & 0,24 & 8,47 & $<0,001$ & 0,18 & 6,39 & $<0,001$ \\
\hline Neuroticismo & $-0,20$ & $-6,94$ & $<0,001$ & $-0,16$ & $-5,60$ & $<0,001$ \\
\hline Abertura & 0,21 & 7,33 & $<0,001$ & 0,20 & 7,19 & $<0,001$ \\
\hline Gratidão & & & & 0,31 & 10,3 & $<0,001$ \\
\hline$R^{2}$ & & 0,35 & & & 0,42 & \\
\hline$R^{2}$ ajustado & & 0,35 & & & 0,42 & \\
\hline$F$ & & $95,1^{*}$ & & & 106,7 & \\
\hline
\end{tabular}

Nota. $N=883$. Regressão linear hierárquica, método Enter, fatores de personalidade no primeiro bloco e gratidão incluída no segundo bloco. ${ }^{* * *} \mathrm{p}<0,001$

Tabela 5

Personalidade e Gratidão como Preditoras do Afeto Negativo.

\begin{tabular}{|c|c|c|c|c|c|c|}
\hline & \multicolumn{3}{|c|}{ Bloco 1} & \multicolumn{3}{|c|}{ Bloco 2} \\
\hline & $\beta$ & $t$ & $p$ & $\beta$ & $t$ & $p$ \\
\hline Constante & & 8,33 & $<0,001$ & & 9,78 & $<0,001$ \\
\hline Extroversão & $-0,08$ & $-2,59$ & 0,010 & $-0,05$ & $-1,50$ & 0,13 \\
\hline Socialização & $-0,08$ & $-2,36$ & 0,019 & $-0,04$ & $-1,33$ & 0,18 \\
\hline Realização & $-0,10$ & $-3,72$ & $<0,001$ & $-0,07$ & $-2,50$ & 0,012 \\
\hline Neuroticismo & 0,57 & 20,28 & $<0,001$ & 0,55 & 19,48 & $<0,001$ \\
\hline Abertura & 0,04 & 1,42 & 0,16 & 0,05 & 1,71 & 0,087 \\
\hline Gratidão & & & & $-0,16$ & $-5,17$ & $<0,001$ \\
\hline$R^{2}$ & \multicolumn{3}{|c|}{0,41} & \multicolumn{3}{|c|}{0,43} \\
\hline$R^{2}$ ajustado & \multicolumn{3}{|c|}{0,41} & \multicolumn{3}{|c|}{0,43} \\
\hline$F$ & \multicolumn{3}{|c|}{$123,4^{* * *}$} & \multicolumn{3}{|c|}{$110,3^{* * *}$} \\
\hline
\end{tabular}

Nota. $N=883$. Regressão linear hierárquica, método Enter, fatores de personalidade no primeiro bloco e gratidão incluída no segundo bloco. ${ }^{* * *} \mathrm{p}<0,001$

\section{Discussão}

Este estudo apresentou evidências de validade de uma versão adaptada do Gratitude Questionnaire-6 (GQ6) para o contexto brasileiro, incluindo-se relações entre gratidão e outras variáveis e o poder preditivo da gratidão sobre o bem-estar subjetivo. Esse instrumento afere o nível de disposição dos indivíduos a sentirem gratidão, compreendendo o construto como um traço afetivo (McCullough et al., 2002). Procedimentos controlados de tradução do inglês para o português do Brasil e de revisão da redação foram adotados a fim de garantir que o conteúdo dos itens mantivesse correspondência com o instrumento original e com a teoria do construto. Análises fatoriais confirmatórias mostraram adequados índices de ajuste para a estrutura de um fator, tal como encontrado no estudo original do instrumento (McCullough et al.,
2002) e em outros estudos de adaptação (e.g., Caputo, 2016; Chen et al., 2009; Gouveia et al., 2019; Kong et al., 2017; Langer et al., 2016). O instrumento ainda apresentou coeficientes alfa e ômega de 0,79 , que podem ser considerados satisfatórios índices de fidedignidade (Nunnally, 1978). Mesmo nos limites inferiores do intervalo de confiança de $95 \%$ para o alfa e ômega o instrumento apresenta satisfatórios índices de consistência interna (Dunn, Baguley, \& Brunsden, 2014).

No primeiro modelo testado, a fim de buscar evidências de validade baseadas na estrutura, sem correlacionar os resíduos dos erros, os valores de CFI, TLI, GFI ficaram próximos de 0,95 , o que pode ser considerado adequado ajuste (ver $\mathrm{Hu} \&$ Bentler, 1999; Hooper, Coughlan, \& Mullen, 2008). Contudo, o valor do RMSEA ficou acima do recomendado (recomendado $=0,06$; observado $=0,094$ ). Ao se correlacionarem os 
resíduos dos itens 3 e 6 , todos os indicadores de ajuste mostraram-se adequados. Esses dois itens são os únicos do instrumento com formato de resposta invertida. Ao se tomarem esses resultados em conjunto, e levando-se em conta a teoria sobre o construto e os estudos anteriores sobre o instrumento, pode-se considerar que esta versão brasileira apresenta satisfatórias evidências de validade baseadas na estrutura.

A análise do instrumento, por meio da Teoria de Resposta ao Item, mostrou que os itens da escala apresentam índice de discriminação moderado (itens 5 e 6) e muito alto (demais itens), segundo classificação de Baker (2001). Apesar do item 6 apresentar o menor parâmetro de discriminação, esse item cobre os níveis mais elevados de gratidão (com as respostas invertidas), ou seja, para endossar o ponto mais extremo da escala de resposta os participantes precisam ter níveis altos de gratidão. Considerando-se os parâmetros de dificuldade, pode-se afirmar que os itens conseguem distinguir, mais eficazmente, os níveis de gratidão em torno de $-4,45$ a 0,69 . Isso significa que essa escala não consegue diferenciar adequadamente níveis elevados do traço latente. Observando-se a curva de informação do teste, nota-se que o erro padrão da medida aumenta a medida que o traço latente atinge valores acima de zero. Versões revisadas do instrumento devem incluir itens mais difíceis (que exijam altos níveis de gratidão para serem endossados), a fim de resolver esse problema.

No que diz respeito às evidências de validade baseadas nas relações com outras variáveis, verificaram-se correlações e diferenças entre grupos de acordo com o esperado. A gratidão correlacionou-se positivamente com autoestima, otimismo, satisfação de vida, afeto positivo $\mathrm{e}$, negativamente, com afeto negativo, tal como tem sido encontrado em outros estudos sobre o construto (e.g., Emmons \& Shelton, 2002; Lin, 2015; Nezlek, Newman, \& Thrash, 2016; Park et al., 2004). As correlações encontradas permitem concluir que quanto mais dispostas as pessoas estão a sentir gratidão, maiores seus níveis de bem-estar, autoestima e otimismo. Esses resultados reforçam a importância da gratidão enquanto um caminho para atingir o bem-estar, tal como tem sido proposto em intervenções (e.g., Emmons \& McCullough, 2003; Froh, Sefick, \& Emmons, 2008).

Ainda, verificaram-se correlações entre gratidão e os fatores de personalidade em acordo com outros estudos (e.g., McCullough et al., 2004; Wood et al., 2008; Wood et al., 2010). As correlações mais fortes foram observadas para os fatores socialização e extroversão, ambas positivas. O contato com outras pessoas e o estabelecimento de relacionamentos amistosos podem estar atuando como facilitadores de situações que permitam sentir gratidão. Também foi verificada uma correlação positiva entre gratidão e idade, tal como em estudos anteriores (Chopik et al., 2019). Pode-se supor que pessoas mais velhas tenham tido mais oportunidades de exercer a gratidão e isso pode ter lhes ensinado a serem gratos em diversas situações.

No que diz respeito às diferenças de médias em gratidão, o instrumento foi capaz de discriminar pessoas de acordo com o esperado teoricamente. As pessoas que tinham uma crença religiosa apresentaram médias mais elevadas de gratidão. A gratidão tem se mostrado positivamente relacionada a uma maior transcendência espiritual, religiosidade e espiritualidade em diversos estudos (e.g., Adler \& Fagley, 2005; Lambert et al., 2009; Kraus et al., 2015; McCullough et al., 2002; McCullough et al., 2004). Esse resultado pode ser consequência do fato de muitas religiões no mundo valorizarem a gratidão e a considerarem uma característica humana desejável (Emmons, 2005), fazendo com que pessoas ligadas à espiritualidade ou religiosas adotem uma perspectiva maior de gratidão e a pratiquem com mais frequência. Ainda, neste estudo as mulheres mostraram maiores níveis de gratidão do que os homens, tal como esperado teoricamente e encontrado em outros estudos (Kashdan et al., 2009). Uma possível explicação para essa diferença pode ser a maior tendência das mulheres a reconhecerem atos benevolentes por parte de outras pessoas e uma facilidade maior em expressar sua apreciação (Kashdan et al., 2009). Essas diferenças se mantiveram mesmo depois de controlados os efeitos da personalidade, ainda, não foi observada interação entre sexo e crença religiosa, indicando que as mulheres tendem a ser mais gratas do que os homens, independentemente da religiosidade e da personalidade; e as pessoas religiosas tendem a ser mais gratas do que as não religiosas, independentemente do sexo e da personalidade.

Por fim, testou-se o poder preditivo da gratidão sobre o bem-estar subjetivo para além do explicado pelos fatores de personalidade. A gratidão mostrou-se variável significativa nos modelos explicativos de todos os componentes do bem-estar. Para a satisfação de vida, $13 \%$ da variância foi explicada pela gratidão e $23 \%$ pelos cinco grandes fatores; para afeto positivo, $7 \%$ da variância foi explicada pela gratidão e $35 \%$ pelos fatores de personalidade; para afeto negativo, $2 \%$ da variância foi explicada pela gratidão e $41 \%$ pelos cinco grandes fatores. Esses resultados são compatíveis com os encontrados em outros estudos e reforçam a independência da gratidão dos cinco grandes fatores de personalidade (McCullough et al., 2002; Wood et al., 2008; Wood, Maltby et al., 2008). Incrementos em gratidão relacionam-se a aumentos de bem-estar subjetivo, principalmente, do aspecto cognitivo do bem-estar.

Os resultados encontrados neste estudo permitem concluir que a versão adaptada para o Brasil do Gratitude Questionnaire - GQ-6 apresenta satisfatórias evidências de validade e adequados índices de fidedignidade. $\mathrm{O}$ uso do GQ-6 para mensurar o nível disposicional de gratidão pode contribuir em pesquisas futuras, por exemplo, que investiguem as relações de gratidão com outras variáveis, os impactos de intervenções em psicologia positiva, a 
predição de comportamentos, etc. Contudo, é importante apontar algumas limitações deste estudo, uma vez que a amostra não é representativa da população brasileira, dada a alta escolaridade e predominância da cor/raça branca dos participantes. Isso implica um instrumento com evidências de validade para uma população específica e, consequentemente, novos estudos devem ser conduzidos com outras amostras.

\section{Referências}

Adler, M. G., \& Fagley, N. S. (2005). Appreciation: Individual differences in finding value and meaning as a unique predictor of subjective well-being. Journal of Personality, 73(1), 79-114. doi: 10.1111/j.1467-6494.2004.00305.x

Allemand, M., \& Hill, P. L. (2016). Gratitude from early adulthood to old age. Journal of Personality, 84(1), 21-35. doi: I: 10.1111/jopy.12134

American Educational Research Association [AERA], American Psychological Association [APA], \& National Council on Measurement in Education [NCME] (2014). Standards for educational and psychological testing. Washington, DC: American Educational Research Association Publications.

Baker, F. B. (2001). The basics of item response theory (2nd ed.). University of Maryland, College Park, MD: ERIC Clearinghouse on Assessment and Evaluation.

Bastianello, M. R., \& Pacico, J. C. (2014). Otimismo. Em Avaliação em psicologia positiva (pp. 95-100). Porto Alegre, RS: Artmed.

Caputo, A. (2016). Italian translation and validation of the Gratitude Questionnaire (GQ-6). International Journal of Wellbeing, 6(2), 80-92. doi:10.5502/ijw.v6i2.492

Chalmers, R. P. (2012). Mirt: A multidimensional item response theory package for the R environment. Journal of Statistical Software, 48(6), 1-29. doi:10.18637/jss.v048.i06

Chen, L. H., Chen, M.-Y., Kee, Y. H., \& Tsai, Y.-M. (2009). Validation of the gratitude questionnaire (GQ) in Taiwanese undergraduate students. Journal of Happiness Study, 10(6), 655-664. doi: 10.1007/s10902-008-9112-7

Chopik, W. J., Newton, N. J., Ryan, L. H., Kashdan, T. B., \& Jarden, A. J. (2019) Gratitude across the life span: Age differences and links to subjective wellbeing. The Journal of Positive Psychology, 14(3), 292-302. doi: 10.1080/17439760.2017.1414296

Diener, E., Emmons, R. A., Larsen, R. J., \& Griffin, S. (1985). The Satisfaction with Life Scale. Journal of Personality Assessment, $49(1), 71-75$. doi: 10.1207/s15327752jpa4901_13

Diener, E., Oishi, S., \& Tay, L. (2018). Advances in subjective well-being research. Nature Human Behavior, 2(4), 253-260. doi: 10.1038/ s41562-018-0307-6

Dunn, T. J., Baguley, T., \& Brunsden, V. (2014). From alpha to omega: A practical solution to the pervasive problem of internal consistency estimation. British Journal of Psychology, 105(3), 399-412. doi: 10.1111/bjop.12046

Emmons, R. A. (2005). Giving thanks: Spiritual and religious correlates of gratitude. Journal of Psychology and Christianity, 24(2), 140-148. Recuperado de http://www.psychology.hku.hk/ftbcstudies/refbase/docs/emmons/2005/57_Emmons +Kneezel2005.pdf

Emmons, R. A., \& McCullough, M. E. (2003). Counting blessings versus burdens: An experimental investigation of gratitude and subjective well-being in daily life. Journal of Personality and Social Psychology, 84(2), 377-389. doi: 10.1037/0022-3514.84.2.377

Emmons, R. A., \& Shelton, C. M. (2002). Gratitude and the science of positive psychology. Em C. R. Snyder \& S. J. Lopez (Eds.), The handbook of positive psychology (pp. 459-471). New York, NY: Oxford University Press.

Froh, J. J., Sefick, W. J., \& Emmons, R. A. (2008). Counting blessings in early adolescents: An experimental study of gratitude and subjective well-being. Journal of School Psychology, 46(2), 213-233. doi:10.1016/j.jsp.2007.03.005

Gouveia, V. V., Ribeiro, M. G. C., Aquino, T. A. A., Loureto, G. D. L., Nascimento, B. S., \& Rezende, A. T. (2019). Gratitude Questionnaire (GQ-6): Evidence of construct validity in Brazil. Current Psychology. doi: 10.1007/s12144-019-00197-x

Hooper, D., Coughlan, J., \& Mullen, M. (2008). Structural equation modelling: Guidelines for determining model fit. Electronic Journal of Business Research Methods, 6(1), 53-60. doi: 10.21427/D7CF7R

Hu, L., \& Bentler, P. M. (1999). Cutoff criteria for fit indexes in covariance structure analysis: Conventional criteria versus new alternatives. Structural Equation Modeling, 6, 1-55. doi: 10.1080/10705519909540118

Hutz, C. S., \& Zanon, C. (2011). Revisão da adaptação, validação e normatização da escala de autoestima de Rosenberg. Avaliação Psicológica, 10(1), 41-49.

Hutz, C. S., Zanon, C., \& Bardagi, M. (2014). Satisfação de Vida. Em C. S. Hutz (Ed.), Avaliação em Psicologia Positiva (pp. 43-48). Porto Alegre: Artmed.

Kashdan, T. B., Mishra, A., Breen, W. E., \& Froh, J. J. (2009). Gender differences in gratitude: Examining appraisals, narratives, the willingness to express emotions, and changes in psychological needs. Journal of Personality, 77(3), 691-730. doi: 10.1111/j.1467-6494.2009.00562.x.

Kelley, K. (2019). MBESS: The MBESS R Package. R package version 4.6.0.

Kong, F., You, X., \& Zhao, J. (2017). Evaluation of the gratitude questionnaire in a Chinese sample of adults: Factorial validity, criterionrelated validity, and measurement invariance across sex. Frontiers in Psychology, 8(1496), 14-98. doi: 10.3389/fpsyg.2017.01498

Kraus, R., Desmond, S. A., \& Palmer, Z. D. (2015). Being thankful: Examining the relationship between young adult religiosity and gratitude. Journal of Religion and Health, 54(4), 1331-1344. doi: 10.1007/s10943-014-9923-2

Krause, N. (2009). Religious involvement, gratitude, and change in depressive symptoms over time. International Journal for the Psychology of Religion, 19(3), 155-172. doi: 10.1080/10508610902880204

Lambert, N. M., Fincham, F. D., Braithwaite, S. R., Graham, S. M., \& Beach, S. R. H. (2009). Can prayer increase gratitude? Psychology of Religion and Spirituality, 1(3), 139-149. doi: 10.1037/a0016731

Langer, Á. I., Ulloa, V. G., Aguilar-Parra, J. M., Araya-Véliz, C., \& Brito, G. (2016). Validation of a Spanish translation of the Gratitude Questionnaire (GQ-6) with a Chilean sample of adults and high schoolers. Health and Quality of Life Outcomes, 14(1), 53. doi: 10.1186/ s12955-016-0450-6 
Lin, C. C. (2015). Gratitude and depression in young adults: The mediating role of self-esteem and well-being. Personality and Individual Differences, 87(2015), 30-34. doi: 10.1016/j.paid.2015.07.017

McCullough, M. E., Emmons, R. A., \& Tsang, J. A. (2002). The grateful disposition: A conceptual and empirical topography. Journal of Personality and Social Psychology, 82(1), 112. doi: 10.1037//0022-3514.82.1.112

McCullough, M. E., Tsang, J. -A., \& Emmons, R. A. (2004). Gratitude in intermediate affective terrain: Links of grateful moods to individual differences and daily emotional experience. Journal of Personality and Social Psychology, 86(2), 295-309. doi: 10.1037/0022-3514.86.2.295

Naito, T., Wangwan, J., \& Tani, M. (2005). Gratitude in university students in Japan and Thailand. Journal of Cross-Cultural Psychology, 36(2), 247-263. doi: 10.1177/0022022104272904

Natividade, J. C. (2018, Junho). A Gratidão no contexto brasileiro: Relações com otimismo, autoestima e bem-estar subjetivo. Em A. C. S. Vazquez et al. (Orgs) III Congresso Brasileiro de Psicologia Positiva, São Paulo, SP.

Natividade, J. C., \& Hutz, C. S. (2015). Escala reduzida de descritores dos cinco grandes fatores de personalidade: prós e contras. Psico, 46(1), 79-89. doi: 10.15448/1980-8623.2015.1.16901

Nezlek, J. B., Newman, D. B., \& Thrash, T. M. (2016). A daily diary study of relationships between feelings of gratitude and well-being. The Journal of Positive Psychology, 12(4), 323-332. doi:10.1080/17439760.2016.1198923

Nunnally, J. C. (1978). Psychometric theory. New York: McGraw Hill.

Paludo, S. S. (2008). Emoções morais e gratidão: Uma nova perspectiva sobre o desenvolvimento de jovens em situação de risco pessoal e social (Tese de doutorado). Universidade Federal do Rio Grande do Sul, Porto Alegre, Brasil. Recuperado de https://www.lume.ufrgs.br/ handle/10183/14289

Park, N., Peterson, C., \& Seligman, M. E. (2004). Strengths of character and well-being. Journal of Social and Clinical Psychology, 23 (5), 603-619. doi: 10.1521/jscp.23.5.603.50748

Park, N., Peterson, C., \& Seligman, M. E. P. (2004). Strengths of character and well-being. Journal of Social \& Clinical Psychology, $23(5), 603-619$. doi: $10.1521 /$ jscp.23.5.603.50748

R Core Team (2019). R: A language and environment for statistical computing. R Foundation for Statistical Computing, Vienna, Austria. Recuperado de https://www.R-project.org/

Rosenberg, M. (1965). The Measurement of Self-Esteem. Em M. Rosenberg (Ed.), Society and the Adolescent Self-Image (pp. 16-36). Princeton University Press.

Rosseel, Y. (2012). lavaan: An R Package for Structural Equation Modeling. Journal of Statistical Software, 48(2), 1-36. Recuperado de http:// www.jstatsoft.org/v48/i02/

Samejima, F. (1969). Estimation of latent ability using a response pattern of graded scores (Psychometric Monograph No. 17). Richmond, VA: Psychometric Society.

Scheier, M. F., Carver, C. S., \& Bridges, M. W. (1994). Distinguishing optimism from neuroticism (and trait anxiety, self-mastery, and selfesteem): A reevaluation of the Life Orientation Test. Journal of Personality and Social Psychology, 67(6), 1063-1078. doi: 10.1037/00223514.67.6.1063

Simon, R. W., \& Nath, L. E. (2004). Gender and emotion in the United States: Do men and women differ in self reports of feelings or expressive behavior? American Journal of Sociology, 109(5), 1137-1176. doi: 10.1086/382111

Wachelke, J., Natividade, J. C., Andrade, A. D., Wolter, R., \& Camargo, B. (2014). Caracterização e avaliação de um procedimento de coleta de dados online (CORP). Avaliação Psicológica, 13(1), 143-146.

Watson, D., Clark, L. A., \& Tellegen, A. (1988). Development and validation of brief measures of positive and negative affect: The PANAS scales. Journal of Personality and Social Psychology, 54(6), 1063-1070. doi.org/10.1037/0022-3514.54.6.1063

Wood, A. M., Froh, J. J., \& Geraghty, A. W. A. (2010). Gratitude and well-being: A review and theoretical integration. Clinical Psychology Review, 30(7), 890-905. doi: 10.1016/j.cpr.2010.03.00

Wood, A. M., Joseph, S., \& Maltby, J. (2008). Gratitude uniquely predicts satisfaction with life: Incremental validity above the domains and facets of the five factor model. Personality and Individual Differences, 45(1), 49-54. doi: 10.1016/j.paid.2008.02.019

Wood, A. M., Joseph, S., \& Maltby, J. (2009). Gratitude predicts psychological well-being above the big five facets. Personality and Individual Differences, 46(4), 443-447. doi:10.1016/j.paid.2008.11.012

Wood, A. M., Maltby, J., Gillett, R., Linley, P. A., \& Joseph, S. (2008). The role of gratitude in the development of social support, stress, and depression: Two longitudinal studies. Journal of Research in Personality, 42(4), 854-871. doi:10.1016/j.jrp.2007.11.003

Wood, A. M., Maltby, J., Stewart, N., \& Joseph, S. (2008). Conceptualizing gratitude and appreciation as a unitary personality trait. Personality and Individual Differences, 44(3), 619-630. doi:10.1016/j.paid.2007.09.028

Woyciekoski, C., Natividade, J. C., \& Hutz, C. S. (2014). As contribuições da personalidade e dos eventos de vida para o bem-estar subjetivo. Psicologia: Teoria e Pesquisa, 30(4), 401-409. doi: 10.1590/s0102-37722014000400005

Zanon, C., \& Hutz, C. S (2014). Escala de afetos positivos e negativos (PANAS). Em C. S. Hutz (Ed.), Avaliação em Psicologia Positiva (pp. 63-69). Porto Alegre: Artmed.

\section{Sobre os autores}

Jean C. Natividade é doutor em Psicologia e professor do Programa de Pós-Graduação em Psicologia da PUC-Rio.

Amanda Londero-Santos é mestre em Psicologia pela Università Degli Studi di Padova e doutoranda do Programa de Pós-Graduação em Psicologia da PUC-Rio.

Nathalia M. Carvalho e Thainá F. Carvalho são mestrandas do Programa de Pós-Graduação em Psicologia da PUC-Rio.

Leonardo S. Santos é psicólogo pela PUC-Rio.

Luiza S. Fagundes é mestre em Psicologia pelo Programa de Pós-Graduação em Psicologia da PUC-Rio. 